This item was submitted to Loughborough's Research Repository by the author.

Items in Figshare are protected by copyright, with all rights reserved, unless otherwise indicated.

\title{
Memory effect in a molecular quantum dot with strong electron-vibron
} interaction

PLEASE CITE THE PUBLISHED VERSION

PUBLISHER

(C) American Physical Society

LICENCE

CC BY-NC-ND 4.0

REPOSITORY RECORD

Alexandrov, A.S., and A.M. Bratkovsky. 2019. "Memory Effect in a Molecular Quantum Dot with Strong Electron-vibron Interaction". figshare. https://hdl.handle.net/2134/1322. 


\title{
Memory effect in a molecular quantum dot with strong electron-vibron interaction
}

\author{
A. S. Alexandrov ${ }^{1,2}$ and A. M. Bratkovsky ${ }^{1}$ \\ ${ }^{1}$ Hewlett-Packard Laboratories, 1501 Page Mill Road, 1L, Palo Alto, California 94304, USA \\ ${ }^{2}$ Department of Physics, Loughborough University, Loughborough LE11 3TU, United Kingdom
}

(Received 17 December 2002; published 11 June 2003)

\begin{abstract}
Polaron theory of tunneling through a molecular quantum dot (MQD) with strong electron-vibron interactions and attractive electron-electron correlations is developed. The dot is modeled as a $d$-fold-degenerate energy level weakly coupled to the leads. The effective attractive interaction between polarons in the dot results in a "switching" phenomenon in the current-voltage characteristics when $d>2$, in agreement with the results for the phenomenological negative- $U$ model. The degenerate MQD with strong electron-vibron coupling has two stable current states in a certain interval of the bias voltage below some critical temperature.

DOI: 10.1103/PhysRevB.67.235312

PACS number(s): 73.63.Nm, 71.38.Mx, 72.10.Fk, 21.45.+v
\end{abstract}

\section{INTRODUCTION}

Strongly correlated transport through mesoscopic systems with repulsive electron-electron interactions has received considerable interest in the past (see, for example (Refs. $1-8$ ), and continues to be the focus of intense experimental and theoretical investigation. ${ }^{9,10}$ The Coulomb interaction suppresses tunneling for a certain range of applied voltages, leading to what is commonly called the Coulomb blockade. There is now growing interest in molecular nanowires and quantum dots used as "transmission lines" 11,12 and active molecular elements ${ }^{13-17}$ in molecular-scale electronics. ${ }^{13}$

A few experimental studies ${ }^{14}$ provide evidence for a molecular switching effect, when the current-voltage (I-V) characteristics show two branches with high and low current for the same voltage. The effect exists in simple molecules, too. ${ }^{18}$ It is important to identify the actual mechanism of switching.

Recently we have proposed a negative- $U$ Hubbard model of a $d$-fold-degenerate quantum dot, with an intrinsic nonretarded switching mechanism when $d>2 .{ }^{19}$ We argued that the attractive electron correlations could be caused by a strong electron-phonon (vibron) interaction in the molecule, and/or by the valence fluctuations.

It has been experimentally demonstrated that the low-bias conductance of molecules is dominated by resonant tunneling through coupled electronic and vibration levels. ${ }^{20}$ Conductance peaks due to electron-vibron interactions have been seen in $\mathrm{C}_{60}(d=6) .{ }^{9}$ Different aspects of the electronphonon/vibron (e-ph) interaction effect on the tunneling through molecules and quantum dots (QD's) have been studied by several authors. ${ }^{21-28}$ In particular, Glazman and Shekhter, and later Wingreen et al., ${ }^{21}$ presented the exact resonant-tunneling transmission probability fully taking into account the e-ph interaction on a nondegenerate resonant site. Phonons produced transmission sidebands but did not affect the integral transmission probability. $\mathrm{Li}$, Chen, and $\mathrm{Zhou}^{22}$ studied the conductance of a double-degenerate (due to spin) quantum dot with Coulomb repulsion and the e-ph interaction. Their numerical results also showed the sideband peaks and the main peak related to the Coulomb repulsion, which was decreased by the e-ph interaction. Kang ${ }^{23}$ studied boson- (vibron-) assisted transport through a double- degenerate QD coupled to two superconducting leads and found multiple peaks in the I-V curves, which originated from the singular BCS density of states and the phonon sidebands. Ermakov ${ }^{24}$ calculated the I-V curves of a fourfolddegenerate dot including both the on-site Coulomb and e-ph interactions. He obtained a switching effect in the numerical I-V curves, similar to that in the negative- $U$ Hubbard model discussed by us recently. ${ }^{19}$ However, using a Hamiltonian averaged over the phonons, Ermakov missed all phonon sidebands and obtained an unphysical population $(n=1)$ of each QD state. More recently Gogolin and Komnik ${ }^{28}$ analyzed a nondegenerate $\mathrm{QD}, d=1$, coupled with a single phonon mode. They found a switching effect in the BornOppenheimer approximation similar to that in our negative$U$ Hubbard model, but surprisingly for a nondegenerate case with $d=1$. However, we have to mention that the BornOppenheimer approximation does not apply to a nondegenerate level, since there are no "fast" (compared to the characteristic phonon time $1 / \omega_{0}$ ) electron transitions within the dot. Despite differences in the models and approximations, Refs. 19, 24, and 28 pointed to a different mechanism of the volatile molecular memory caused by the e-ph or any other attractive electron correlations.

Here we develop the analytical theory of correlated transport through a degenerate molecular quantum dot (MQD) fully taking into account both Coulomb and e-ph interactions. We show that the phonon sidebands significantly modify the switching behavior of the I-V curves in comparison with the negative- $U$ Hubbard model. ${ }^{19}$ Nevertheless, the switching effect is robust. It appears when the effective interaction of polarons is attractive and the state of the dot is multiply degenerate, $d>2$.

\section{STEADY CURRENT THROUGH MQD's}

We apply the Landauer-type expression for the steady current through a region of interacting electrons, derived by Meir and Wingreen ${ }^{7}$ as (in units $\hbar=k_{B}=1$ )

$$
I(V)=-\frac{e}{\pi} \int_{-\infty}^{\infty} d \omega\left[f_{1}(\omega)-f_{2}(\omega)\right] \operatorname{Im} \operatorname{Tr}\left[\hat{\Gamma}(\omega) \hat{G}^{R}(\omega)\right],
$$




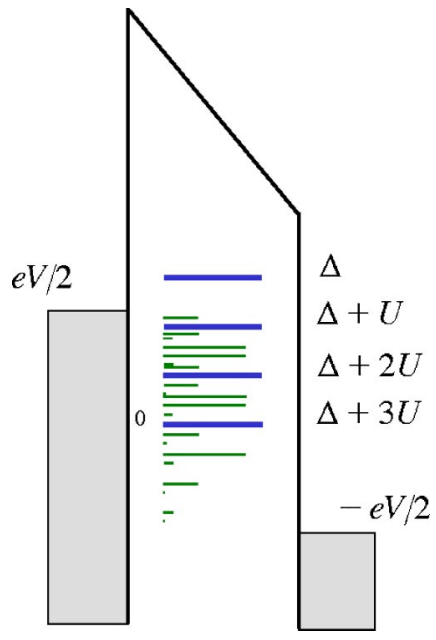

FIG. 1. (Color online) Schematic of the energy levels and phonon sidebands for a molecular quantum dot under bias voltage $V$ $(e V / 2 \Delta=0.75)$ with the coupling constant $\gamma^{2}=11 / 13$ and total electron-electron correlation energy $U<0$, Eq. (10). The level is assumed to be fourfold degenerate $(d=4)$ with energies $\Delta+r U$, $r=0, \ldots,(d-1)$ (thick bars). Thin bars show the vibron sidebands with the size of the bar proportional to the weight of the particular contribution in the density of states (see text) in the case of one vibron with frequency $\omega_{0} / \Delta=0.2$ at $T=0$. Only the bands in the energy window $(e V / 2,-e V / 2)$ (shown) contribute to current at zero temperature.

where $f_{1(2)}(\omega)=\left\{\exp \left[\left(\omega+\Delta_{(+)}^{-} e V / 2\right) / T\right]+1\right\}^{-1}, \quad T$ is the temperature, and $\Delta$ is the position of the lowest unoccupied molecular level with respect to the chemical potential. $\widehat{\Gamma}(\omega)$ depends on the density of states (DOS) in the leads and on the hopping integrals connecting one-particle states in the left (1) and the right (2) leads with the states in the MQD, Fig. 1. This formula includes, by means of the Fourier transform of the full molecular retarded Green's function (GF), $\hat{G}^{R}(\omega)$, the e-ph and Coulomb interactions inside the MQD and coupling to the leads. Since the leads are metallic, electron-electron and e-ph interactions in the leads, and interactions of electrons in the leads with electrons and phonons in the MQD can be neglected. We are interested in the tunneling near the conventional threshold, $e V=2 \Delta$, Fig. 1 , within a voltage range about an effective attractive potential $|U|$ caused by phonons/vibrons (see below).

The attractive energy is the difference of two large interactions, the Coulomb repulsion and the phonon-mediated attraction, of the order of $1 \mathrm{eV}$ each. Hence, $|U|$ is on the order of a few tenths of one $\mathrm{eV}$. We neglect the energy dependence of $\hat{\Gamma}(\omega) \approx \Gamma$ at this scale, and assume that the coupling to the leads is weak, $\Gamma \ll|U|$. In this case $\hat{G}^{R}(\omega)$ does not depend on the leads. Moreover, we assume that there is a complete set of one-particle molecular states $|\mu\rangle$, where $\hat{G}^{R}(\omega)$ is diagonal. With these assumptions we can reduce Eq. (1) to

$$
I(V)=I_{0} \int_{-\infty}^{\infty} d \omega\left[f_{1}(\omega)-f_{2}(\omega)\right] \rho(\omega)
$$

allowing for a transparent analysis of essential physics of the switching phenomenon. Here $I_{0}=e \Gamma$ and the molecular DOS, $\rho(\omega)$, is given by

$$
\rho(\omega)=-\frac{1}{\pi} \sum_{\mu} \operatorname{Im} \hat{G}_{\mu}^{R}(\omega),
$$

where $\hat{G}_{\mu}^{R}(\omega)$ is the Fourier transform of $\hat{G}_{\mu}^{R}(t)=-i \theta(t)$ $\times\left\langle\left\{c_{\mu}(t), c_{\mu}^{\dagger}\right\}\right\rangle,\{\cdots, \cdots\}$ is the anticommutator, $c_{\mu}(t)$ $=e^{i H t} c_{\mu} e^{-i H t}$, and $\theta(t)=1$ for $t>0$ and zero otherwise. We calculate $\rho(\omega)$ exactly in Sec. III in the framework of the Hamiltonian, which includes both the Coulomb $U^{C}$ and e-ph interactions as

$$
\begin{aligned}
H= & \sum_{\mu} \varepsilon_{\mu} \hat{n}_{\mu}+\frac{1}{2} \sum_{\mu^{\prime} \mu^{\prime}} U_{\mu \mu^{\prime}}^{C} \hat{n}_{\mu} \hat{n}_{\mu^{\prime}}+\sum_{\mu, q} \hat{n}_{\mu} \omega_{q}\left(\gamma_{\mu q} d_{q}+\text { H.c. }\right) \\
& +\sum_{q} \omega_{q}\left(d_{q}^{\dagger} d_{q}+1 / 2\right) .
\end{aligned}
$$

Here $\varepsilon_{\mu}$ are one-particle molecular energy levels; $\hat{n}_{\mu}$ $=c_{\mu}^{\dagger} c_{\mu}$ the occupation number operators, $c_{\mu}$ and $d_{q}$ annihilate electrons and phonons, respectively; $\omega_{q}$ are the phonon (vibron) frequencies; and $\gamma_{\mu q}$ are e-ph coupling constants ( $q$ enumerates the vibron modes). This Hamiltonian conserves the occupation numbers of molecular states $\hat{n}_{\mu}{ }^{29}$ Hence it is compatible with Eq. (2).

\section{MQD DENSITY OF STATES}

We apply the canonical polaron unitary transformation $e^{S},{ }^{30}$ integrating phonons out, as

$$
\widetilde{H}=e^{S} H e^{-S},
$$

where

$$
S=-\sum_{q, \mu} \hat{n}_{\mu}\left[\gamma_{\mu q} d_{q}-\text { H.c. }\right]
$$

is such that $S^{\dagger}=-S$. The electron and phonon operators are transformed as

$$
\tilde{c}_{\mu}=c_{\mu} X_{\mu}
$$

and

$$
\widetilde{d}_{q}=d_{q}-\sum_{\mu} \hat{n}_{\mu} \gamma_{\mu q}^{*},
$$

respectively. Here

$$
X_{\mu}=\exp \left[\sum_{q} \gamma_{\mu q} d_{q}-\text { H.c. }\right] .
$$

The Lang-Firsov canonical transformation shifts ions to new equilibrium positions with no effect on the phonon frequencies. The diagonalization is exact:

$$
\widetilde{H}=\sum_{i} \widetilde{\varepsilon}_{\mu} \hat{n}_{\mu}+\sum_{q} \omega_{q}\left(d_{q}^{\dagger} d_{q}+1 / 2\right)+\frac{1}{2} \sum_{\mu \neq \mu^{\prime}} U_{\mu \mu^{\prime}} \hat{n}_{\mu} \hat{n}_{\mu^{\prime}},
$$

where 


$$
U_{\mu \mu^{\prime}} \equiv U_{\mu \mu^{\prime}}^{C}-2 \sum_{q} \gamma_{\mu q}^{*} \gamma_{\mu^{\prime} q} \omega_{q}
$$

is the interaction of polarons comprising their interaction via molecular deformations (vibrons) and nonvibron (e.g., Coulomb repulsion) $U_{\mu \mu^{\prime}}^{C}$. To simplify the discussion, we shall assume, without losing generality, that the Coulomb integrals do not depend on the orbital index, i.e., $U_{\mu \mu^{\prime}}=U$.

The molecular energy levels are shifted by the polaron level shift due to a deformation well created by the polaron,

$$
\tilde{\varepsilon}_{\mu}=\varepsilon_{\mu}-\sum_{q}\left|\gamma_{\mu q}\right|^{2} \omega_{q}
$$

Applying the same transformation in the retarded GF we obtain

$$
\begin{aligned}
G_{\mu}^{R}(t)= & -i \theta(t)\left\langle\left\{c_{\mu}(t) X_{\mu}(t), \quad c_{\mu}^{\dagger} X_{\mu}^{\dagger}\right\}\right\rangle \\
= & -i \theta(t)\left[\left\langle c_{\mu}(t) c_{\mu}^{\dagger}\right\rangle\left\langle X_{\mu}(t) X_{\mu}^{\dagger}\right\rangle\right. \\
& \left.+\left\langle c_{\mu}^{\dagger} c_{\mu}(t)\right\rangle\left\langle X_{\mu}^{\dagger} X_{\mu}(t)\right\rangle\right],
\end{aligned}
$$

where now electron and phonon operators are averaged over the quantum state of the transformed Hamiltonian $\widetilde{H}$. There is no coupling between polarons and vibrons in the transformed Hamiltonian, and the electron and phonon averages are independent. The Heisenberg phonon operators evolve as

$$
d_{q}(t)=d_{q} e^{-i \omega_{q} t},
$$

so that we find after thermodynamic averaging of the phonon correlator over phonon occupation numbers (using the Weyl's identity for exponential operators)

$$
\begin{aligned}
& \left\langle X_{\mu}(t) X_{\mu}^{\dagger}\right\rangle \\
& \quad=\exp \left\{\sum_{q} \frac{\left|\gamma_{\mu q}\right|^{2}}{\sinh \frac{\beta \omega_{q}}{2}}\left[\cos \left(\omega t+i \frac{\beta \omega_{q}}{2}\right)-\cosh \frac{\beta \omega_{q}}{2}\right]\right\},
\end{aligned}
$$

where $\beta=1 / T$. Repeating the calculations for $\left\langle X_{\mu}^{\dagger} X_{\mu}(t)\right\rangle$ we find a simple useful relation

$$
\left\langle X_{\mu}^{\dagger} X_{\mu}(t)\right\rangle=\left\langle X_{\mu}(t) X_{\mu}^{\dagger}\right\rangle^{*} .
$$

At low temperatures $T \ll \omega_{q}$ the phonon correlator (14) simplifies to

$$
\left\langle X_{\mu}(t) X_{\mu}^{\dagger}\right\rangle=\exp \left[\sum_{\mathbf{q}}\left|\gamma_{\mu q}\right|^{2}\left(e^{-i \omega_{q} t}-1\right)\right] .
$$

Next, we introduce the $N$-particle GFs, which will necessarily appear in the equations of motion for $\left\langle c_{\mu}(t) c_{\mu}^{\dagger}\right\rangle$, as

$$
G_{\mu}^{(N,+)}(t) \equiv-i \theta(t) \sum_{\mu_{1} \neq \mu_{2} \neq \ldots \mu}\left\langle c_{\mu}(t) c_{\mu}^{\dagger} \prod_{i=1}^{N-1} \hat{n}_{\mu_{i}}\right\rangle,
$$

and

$$
G_{\mu}^{(N,-)}(t) \equiv-i \theta(t) \sum_{\mu_{1} \neq \mu_{2} \neq \ldots \mu}\left\langle c_{\mu}^{\dagger} c_{\mu}(t) \prod_{i=1}^{N-1} \hat{n}_{\mu_{i}}\right\rangle .
$$

Then, using the equation of motion for the Heisenberg polaron operator,

$$
i \frac{d c_{\mu}}{d t}=\left[\tilde{\varepsilon}_{\mu}+U \sum_{\mu^{\prime}(\neq \mu)} \hat{n}_{\mu^{\prime}}\right] c_{\mu},
$$

we derive the following equations for the $N$-particle GFs,

$$
\begin{aligned}
i \frac{d G_{\mu}^{(N,+)}(t)}{d t}= & \delta(t)\left(1-n_{\mu}\right) \sum_{\mu_{1} \neq \mu_{2} \neq \ldots \mu} \prod_{i=1}^{N-1} n_{\mu_{i}} \\
& +\left[\tilde{\varepsilon}_{\mu}+(N-1) U\right] G_{\mu}^{(N,+)}(t)+U G_{\mu}^{(N+1,+)}(t),
\end{aligned}
$$

and

$$
\begin{aligned}
i \frac{d G_{\mu}^{(N,-)}(t)}{d t}= & \delta(t) n_{\mu} \sum_{\mu_{1} \neq \mu_{2} \neq \ldots \mu} \prod_{i=1}^{N-1} n_{\mu_{i}} \\
& +\left[\tilde{\varepsilon}_{\mu}+(N-1) U\right] G_{\mu}^{(N,-)}(t)+U G_{\mu}^{(N+1,-)}(t),
\end{aligned}
$$

where $n_{\mu}=\left\langle c_{\mu}^{\dagger} c_{\mu}\right\rangle$ is the expectation number of electrons on the molecular level $\mu$.

We can readily solve this set of coupled equations for MQD with one $d$-fold-degenerate energy level and with the e-ph coupling $\gamma_{\mu q}=\gamma_{q}$, which does not break the degeneracy. At zero-bias voltage the empty level will lie by some energy $\Delta$ above the Fermi levels of the electrodes (Fig. 1). Assuming that $n_{\mu}=n$, Fourier transformation of the set yields for $N=1$

$$
\begin{gathered}
G_{\mu}^{(1,+)}(\omega)=(1-n) \sum_{r=0}^{d-1} \frac{Z_{r}(n)}{\omega-r U+i \delta}, \\
G_{\mu}^{(1,-)}(\omega)=n \sum_{r=0}^{d-1} \frac{Z_{r}(n)}{\omega-r U+i \delta},
\end{gathered}
$$

where $\delta=+0$, and

$$
Z_{r}(n)=\frac{(d-1) !}{r !(d-1-r) !} n^{r}(1-n)^{d-1-r}
$$

\section{A. MQD Green's function at low temperatures $\left(T \ll \omega_{q}\right)$}

It is easiest to find the total Green's function at low temperatures. Indeed, transforming back to real time and using Eqs. (22), (23), and (16), (15) we arrive at 


$$
\begin{aligned}
G_{\mu}^{R}(t)= & -i \theta(t) \mathcal{Z}_{0} \sum_{r=0}^{d-1} Z_{r}(n) e^{-i r U t} \\
& \times\left[(1-n) \exp \left(\sum_{q}\left|\gamma_{\mu q}\right|^{2} e^{-i \omega_{q} t}\right)\right. \\
& \left.+n \exp \left(\sum_{q}\left|\gamma_{\mu q}\right|^{2} e^{i \omega_{q} t}\right)\right],
\end{aligned}
$$

where

$$
\mathcal{Z}_{0}=\exp \left[-\sum_{\mathbf{q}}\left|\gamma_{q}\right|^{2}\right]
$$

is the so-called polaron reduction factor at zero temperature. This is an exact solution, with respect to correlations and e-ph interactions, which satisfies all sum rules. Expanding the exponents in the brackets, we find its Fourier component to be

$$
G_{\mu}^{R}(\omega)=\mathcal{Z}_{0} \sum_{r=0}^{d-1} Z_{r}(n)\left[\frac{1}{\omega-r U+i \delta}+\sum_{l=1}^{\infty} \frac{1}{l !} \sum_{q_{1} \ldots q_{l}}\left|\gamma_{q_{1}} \ldots \gamma_{q_{l}}\right|^{2}\left(\frac{1-n}{\omega-r U-\sum_{k=1}^{l} \omega_{q_{k}}+i \delta}+\frac{n}{\omega-r U+\sum_{k=1}^{l} \omega_{q_{k}}+i \delta}\right)\right]
$$

If the e-ph interaction is weak, $\left|\gamma_{q}\right| \ll 1$, the essential contribution comes only from the first (phononless) term, and we recover the result of the Hubbard model, ${ }^{19}$

$$
G_{\mu}^{R}(\omega)=\sum_{r=0}^{d-1} \frac{Z_{r}(n)}{\omega-r U+i \delta} .
$$

At finite $\left|\gamma_{q}\right| \gtrsim 1$, the phonon sidebands become important in Eq. (27), which is obviously in the form of the multiphonon expansion. If one neglects the correlations, $U=0$, a standard polaron GF (Ref. 31) is recovered:

$$
\begin{aligned}
G_{\mu}^{R}(\omega)= & \mathcal{Z}_{0}\left[\frac{1}{\omega+i \delta}+\sum_{l=1}^{\infty} \frac{1}{l !} \sum_{q_{1} \ldots q_{l}}\left|\gamma_{q_{1}} \ldots \gamma_{q_{l}}\right|^{2}\right. \\
& \left.\times\left(\frac{1-n}{\omega-\sum_{k=1}^{l} \omega_{q_{k}}+i \delta}+\frac{n}{\omega+\sum_{k=1}^{l} \omega_{q_{k}}+i \delta}\right)\right],
\end{aligned}
$$

where we have applied the sum rule $\sum_{r=0}^{d-1} Z_{r}(n)=1$.

\section{B. MQD Green's function at finite temperatures, single vibron mode}

By applying the same method, as in the case of $T=0$, and going over back to real time with the use of Eqs. (22), (23) and (16), (15), we arrive at

$$
\begin{aligned}
G_{\mu}^{R}(t)= & -i \theta(t) \mathcal{Z} \sum_{r=0}^{d-1} Z_{r}(n) e^{-i r U t} \\
& \times\left\{(1-n) \exp \left[\sum_{q} \frac{\left|\gamma_{\mu q}\right|^{2}}{\sinh \frac{\beta \omega_{q}}{2}} \cos \left(\omega t+i \frac{\beta \omega_{q}}{2}\right)\right]\right. \\
& \left.\left.+n \exp \left[\sum_{q} \frac{\left|\gamma_{\mu q}\right|^{2}}{\sinh \frac{\beta \omega_{q}}{2}} \cos \left(\omega t-i \frac{\beta \omega_{q}}{2}\right)\right]\right\}, \quad 30\right)
\end{aligned}
$$

where the polaron reduction factor at finite temperature is

$$
\mathcal{Z}=\exp \left[-\sum_{\mathbf{q}}\left|\gamma_{q}\right|^{2} \operatorname{coth} \frac{\beta \omega_{q}}{2}\right] .
$$

In approximation, where we retain a coupling to a single mode with the characteristic frequency $\omega_{0}$ and $\gamma_{q} \equiv \gamma$, we can expand the exponents in the temporal Green's function (30) in powers of $\exp \left[\omega t+i\left(\beta \omega_{0} / 2\right)\right]$. It is then trivial to find the Green's function in the frequency domain as

$$
\begin{aligned}
G_{\mu}^{R}(\omega)= & \mathcal{Z} \sum_{r=0}^{d-1} Z_{r}(n) \sum_{l=0}^{\infty} I_{l}(\xi) \\
& \times\left[e^{\beta \omega_{0} l / 2}\left(\frac{1-n}{\omega-r U-l \omega_{0}+i \delta}+\frac{n}{\omega-r U+l \omega_{0}+i \delta}\right)\right. \\
& +\left(1-\delta_{l 0}\right) e^{-\beta \omega_{0} l / 2} \\
& \left.\times\left(\frac{1-n}{\omega-r U+l \omega_{0}+i \delta}+\frac{n}{\omega-r U-l \omega_{0}+i \delta}\right)\right],
\end{aligned}
$$

where $\xi=|\gamma|^{2} / \sinh \beta \omega_{0} / 2, I_{l}(\xi)$ is the modified Bessel function, and $\delta_{l k}$ is the Kroneker symbol. At low temperatures, where $\beta \omega_{0} \gg 1, \xi \ll 1$, and $I_{l}(\xi) \approx(\xi / 2)^{l} / l$ ! , this expression gives Eq. (27) in the form 


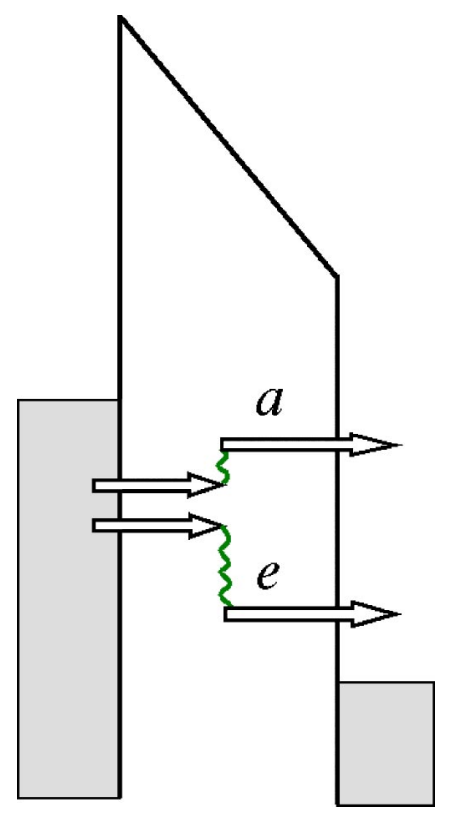

with leads one can apply the Fermi-Dirac golden rule to obtain an equation for $n$. Equating incoming and outgoing numbers of electrons in MQD per unit time we obtain the selfconsistent equation for the level occupation $n$ to be

$$
\begin{aligned}
& (1-n) \int_{-\infty}^{\infty} d \omega\left\{\Gamma_{1} f_{1}(\omega)+\Gamma_{2} f_{2}(\omega)\right\} \rho(\omega) \\
& -n \int_{-\infty}^{\infty} d \omega\left\{\Gamma_{1}\left[1-f_{1}(\omega)\right]+\Gamma_{2}\left[1-f_{2}(\omega)\right]\right\} \rho(\omega)=0,
\end{aligned}
$$

where $\Gamma_{1(2)}$ are the transition rates from left (right) leads to the MQD. Taking into account that $\int_{-\infty}^{\infty} \rho(\omega)=d$, Eq. (35) for the symmetric leads, $\Gamma_{1}=\Gamma_{2}$, reduces to

$$
2 n d=\int d \omega \rho(\omega)\left(f_{1}+f_{2}\right),
$$

which automatically satisfies $0 \leqslant n \leqslant 1$. Explicitly, the selfconsistent equation for the occupation number is

FIG. 2. (Color online) Schematic of the inelastic processes assisting tunneling through the molecular quantum dot (see Fig. 1). The tunneling through the coupled electron-vibron system may proceed with the emission (process $e$ ) or absorption (process $a$ ) of the vibrons. The absorption is possible only at nonzero temperatures.

$$
\begin{aligned}
G_{\mu}^{R}(\omega)= & \mathcal{Z}_{0} \sum_{r=0}^{d-1} Z_{r}(n) \sum_{l=0}^{\infty} \frac{|\gamma|^{2 l}}{l !} \\
& \times\left(\frac{1-n}{\omega-r U-l \omega_{0}+i \delta}+\frac{n}{\omega-r U+l \omega_{0}+i \delta}\right) .
\end{aligned}
$$

The molecular DOS is readily found as an imaginary part of Eq. (32):

$$
\begin{aligned}
\rho(\omega)= & \mathcal{Z} d \sum_{r=0}^{d-1} Z_{r}(n) \sum_{l=0}^{\infty} I_{l}(\xi) \\
& \times\left\{e^{\beta \omega_{0} l / 2}\left[(1-n) \delta\left(\omega-r U-l \omega_{0}\right)+n \delta\left(\omega-r U+l \omega_{0}\right)\right]\right. \\
& +\left(1-\delta_{l 0}\right) e^{-\beta \omega_{0} l / 2}\left[n \delta\left(\omega-r U-l \omega_{0}\right)\right. \\
& \left.\left.+(1-n) \delta\left(\omega-r U+l \omega_{0}\right)\right]\right\}
\end{aligned}
$$

The important feature of the DOS, Eq. (34), is its nonlinear dependence on the occupation number $n$, which leads to the switching effect and hysteresis in the I-V characteristics for $d>2$, as is shown below. It contains full information about all possible correlation and inelastic effects in transport, in particular, all the vibron-assisted tunneling processes (Fig. 2) and phonon sidebands, and describes the renormalization of hopping to the leads.

\section{NONLINEAR RATE EQUATION AND SWITCHING}

Generally, the electron density $n_{\mu}$ obeys an infinite set of rate equations for many-particle GFs which can be derived in the framework of a tunneling Hamiltonian including correlations. ${ }^{19}$ In the case of a MQD only weakly coupled

$$
n=\frac{1}{2} \sum_{r=0}^{d-1} Z_{r}(n)\left[n a_{r}+(1-n) b_{r}\right],
$$

where

$$
\begin{aligned}
a_{r}= & \mathcal{Z} \sum_{l=0}^{\infty} I_{l}(\xi)\left\{e^{\beta \omega_{0} l / 2}\left[f_{1}\left(r U-l \omega_{0}\right)+f_{2}\left(r U-l \omega_{0}\right)\right]\right. \\
& \left.+\left(1-\delta_{l 0}\right) e^{-\left(\beta \omega_{0} l / 2\right)}\left[f_{1}\left(r U+l \omega_{0}\right)+f_{2}\left(r U+l \omega_{0}\right)\right]\right\} \\
b_{r}= & \mathcal{Z} \sum_{l=0}^{\infty} I_{l}(\xi)\left\{e^{\beta \omega_{0} l / 2}\left[f_{1}\left(r U+l \omega_{0}\right)+f_{2}\left(r U+l \omega_{0}\right)\right]\right. \\
& \left.+\left(1-\delta_{l 0}\right) e^{-\left(\beta \omega_{0} l / 2\right)}\left[f_{1}\left(r U-l \omega_{0}\right)+f_{2}\left(r U-l \omega_{0}\right)\right]\right\} .
\end{aligned}
$$

The current is expressed as

$$
j \equiv \frac{I(V)}{d I_{0}}=\sum_{r=0}^{d-1} Z_{r}(n)\left[n a_{r}^{\prime}+(1-n) b_{r}^{\prime}\right],
$$

where

$$
\begin{aligned}
a_{r}^{\prime}= & \mathcal{Z} \sum_{l=0}^{\infty} I_{l}(\xi)\left\{e^{\beta \omega_{0} l / 2}\left[f_{1}\left(r U-l \omega_{0}\right)-f_{2}\left(r U-l \omega_{0}\right)\right]\right. \\
& \left.+\left(1-\delta_{l 0}\right) e^{-\left(\beta \omega_{0} l / 2\right)}\left[f_{1}\left(r U+l \omega_{0}\right)-f_{2}\left(r U+l \omega_{0}\right)\right]\right\}, \\
b_{r}^{\prime}= & \mathcal{Z} \sum_{l=0}^{\infty} I_{l}(\xi)\left\{e^{\beta \omega_{0} l / 2}\left[f_{1}\left(r U+l \omega_{0}\right)-f_{2}\left(r U+l \omega_{0}\right)\right]\right. \\
& \left.+\left(1-\delta_{l 0}\right) e^{-\left(\beta \omega_{0} l / 2\right)}\left[f_{1}\left(r U-l \omega_{0}\right)-f_{2}\left(r U-l \omega_{0}\right)\right]\right\} .
\end{aligned}
$$

Let us analyze the I-V curves for $d=1,2$, and 4 . 


\section{A. Absence of switching for a nondegenerate and twofold- degenerate MQD}

There is one term in the sum over $r, r=0$ with $Z_{0}(n)$ $=1$, if $d=1$. Hence there is only one solution of the rate Eq. (37),

$$
n=\frac{b_{0}}{2+b_{0}-a_{0}},
$$

and the current is single valued at any voltage,

$$
j=\frac{2 b_{0}^{\prime}+a_{0}^{\prime} b_{0}-a_{0} b_{0}^{\prime}}{2+b_{0}-a_{0}} .
$$

This is an exact result, which is valid for any e-ph coupling and any phonon frequency. We have to conclude that there is no switching of a nondegenerate MQD. The opposite conclusion reached in Ref. 28 might be due to the BornOppenheimer (static) approximation used by Gogolin and Komnik. In fact, the Born-Oppenheimer approximation does not apply to the nondegenerate level model, since there are no "fast" (compared to phonon times $1 / \omega_{0}$ ) electron transitions within the "molecule."

In the case of a double-degenerate MQD, $d=2$, there are two terms, which contribute to the sum over $r$, with $Z_{0}(n)$ $=1-n$ and $Z_{1}(n)=n$. The rate equation becomes a quadratic one,

$$
n^{2}\left(a_{0}+a_{1}-b_{0}-b_{1}\right)+n\left(2-a_{0}+b_{0}-b_{1}\right)-b_{0}=0,
$$

with two solutions,

$$
\begin{aligned}
n_{1.2}= & -\frac{2-a_{0}+b_{0}-b_{1}}{2\left(a_{0}+a_{1}-b_{0}-b_{1}\right)} \\
& \pm\left[\frac{\left(2-a_{0}+b_{0}-b_{1}\right)^{2}}{4\left(a_{0}+a_{1}-b_{0}-b_{1}\right)^{2}}+\frac{b_{0}}{a_{0}+a_{1}-b_{0}-b_{1}}\right]^{1 / 2} .
\end{aligned}
$$

However, one of them is negative because $0<b_{r}<a_{r}<1$ for any temperature and voltage. Therefore, we conclude that there is only one physical population of MQD, and the current is also single valued at any voltage and temperature, in agreement with the Hubbard model. ${ }^{19}$

\section{B. Switching of the fourfold-degenerate MQD}

In this case the rate equation is of the fourth power in $n$,

$$
\begin{aligned}
2 n= & (1-n)^{3}\left[n a_{0}+(1-n) b_{0}\right]+3 n(1-n)^{2}\left[n a_{1}+(1-n) b_{1}\right] \\
& +3 n^{2}(1-n)\left[n a_{2}+(1-n) b_{2}\right]+n^{3}\left[n a_{3}+(1-n) b_{3}\right] .
\end{aligned}
$$

In the limit $|\gamma| \ll 1$ we have $b_{r}=a_{r}, \mathcal{Z}=1$, the remaining interaction is $U=U^{C}$ [see Eq. (10)], and Eq. (47) is reduced to

$$
2 n=(1-n)^{3} b_{0}+3 n(1-n)^{2} b_{1}+3 n^{2}(1-n) b_{2}+n^{3} b_{3} .
$$

If we assume now that the nonvibron interaction $U^{C}$ is negative, for example, due to valence fluctuations, then we recover the negative- $U$ model, ${ }^{19}$ and the kinetic equation is reduced to

$$
2 n=1-(1-n)^{3}
$$

in the voltage range $\Delta-|U|<e V / 2<\Delta$, at $T=0$ because $b_{0}=0$ and $b_{1}=b_{2}=b_{3}=1$ there, if $|U|<\Delta / 2$. The current is simplified as

$$
j=2 n \text {. }
$$

Equation (49) has two physical roots, $n=0$ and $n=(3$ $\left.-5^{1 / 2}\right) / 2 \approx 0.38$. Hence we obtain two stationary states of the MQD with low (zero at $T=0$ ) and high current, and $j$ $\approx 0.76$ for the same voltage as we discussed earlier in Ref. 19. The current-voltage characteristics show a hysteretic behavior for $d=4$. When the voltage increases from zero, the fourfold-degenerate MQD remains in a low-current state until the threshold $e V_{2} / 2=\Delta$ is reached. Remarkably, when the voltage decreases from the value above the threshold $V_{2}$, the molecule remains in the high-current state down to the voltage $e V_{1} / 2=\Delta-|U|$ well below the threshold $V_{2}$. This is a correlation mechanism of electronic molecular switching without retardation. Therefore, the negative- $U$ degenerate molecular dot possesses the volatile memory originating from the many-particle attractive correlations. The e-ph coupling results in the phonon sidebands of the DOS, which are fully taken into account in Eq. (35) for the self-consistent occupation of the molecular level $n$. In the case of the Coulomb repulsion and the electron-vibron coupling the effective interaction will be attractive, if

$$
|\gamma| \geqslant\left(\frac{U^{C}}{2 \omega_{0}}\right)^{1 / 2} .
$$

There is an important difference between switching with vibron-mediated electron-electron attraction and the negative- $U$ model. We show the numerical results for $\omega_{0}$ $=0.2$ (in units of $\Delta$, as all the energies in the problem) and $U^{C}=0$ for two values of the coupling constant, $\gamma^{2}=11 / 13$ (Fig. 3) and $\gamma^{2}=13 / 11$ (Fig. 4). This case formally corresponds to $U=-2 \gamma^{2} \omega_{0} \approx-0.4$, i.e., close to the same value of the attraction as we have used in the negative- $U$ model $^{19}$ (we selected those values of $\gamma^{2}$ to avoid accidental commensurability of a ladder of the correlated level energies separated by $U$ and phonon sideband ladders generated by them with the step $\left.\omega_{0}\right)$. In the negative- $U$ case the threshold voltages were $e V_{1} / 2 \Delta=1-0.4=0.6$ and $e V_{2} / 2 \Delta=1$. However, in the vibron case the threshold for the onset of bistability corresponds to a larger voltage bias compared to the negative- $U$ case (at $e V / 2 \Delta=0.86$ for $\gamma^{2}=11 / 13$ and $\omega_{0}$ $=0.2$ ). The $\mathrm{I}-\mathrm{V}$ in the vibron case is much more complex, 


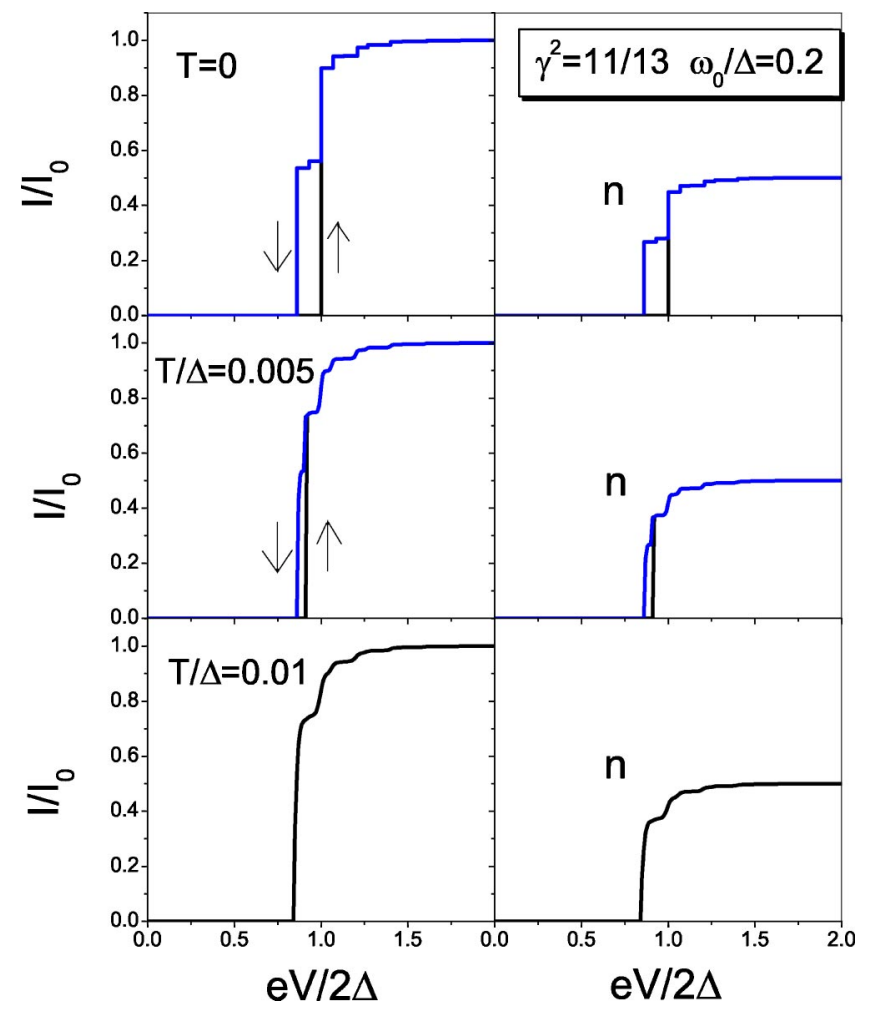

FIG. 3. (Color online) The I-V curves for tunneling through the molecular quantum dot (Fig. 1) with the electron-vibron coupling constant $\gamma^{2}=11 / 13$ and $\omega_{0} / \Delta=0.2$. The up arrows show that the current picks up at some voltage when it is biased, and then drops at lower voltage when the bias is reduced. The bias dependence of current basically repeats the shape of the level occupation $n$ (right column). Steps on the curve correspond to the changing population of the phonon sidebands, which are shown in Fig. 1. The current hysteresis persists up to some critical temperature, which is low, $T / \Delta \approx 0.01$.

too: as one can see from Figs. 3 and 4, the current discontinuity at the threshold strongly depends on the value of the e-ph coupling constant. The inelastic tunneling processes through the level, accompanied by emission/absorption of the phonons (Fig. 2), manifest themselves as steps on the I-V curve, Figs. 3 and 4. Those steps are generated by the phonon sidebands originating from correlated levels on the dot with the energies $\Delta, \Delta+U, \ldots$, and $\Delta+(d-1) U$. Since $\omega_{0}$ is not generally commensurate with $U$, we obtain a fairly irregular picture of the steps on the I-V curve. This comes as no surprise, since kinetic Eq. (47) is much more complex compared to the one in the negative- $U$ case, cf. Eq. (49). The level occupation approaches the limiting value $n=0.5$ at large bias voltages, Figs. 3 and 4.

The bistability region shrinks with temperature. In the specific example of the negative- $U$ model with $U / \Delta$ $=-0.4$ the bistability is over at $T / \Delta \approx 0.1 .{ }^{19}$ Importantly, in the vibron case this happens at much lower temperatures. Indeed, the hysteresis loop almost closes at $T / \Delta=0.01$. The critical temperature, below which the current bistability exists in the vibron case, is suppressed by about an order of magnitude compared to the negative- $U$ case. At finite tem-

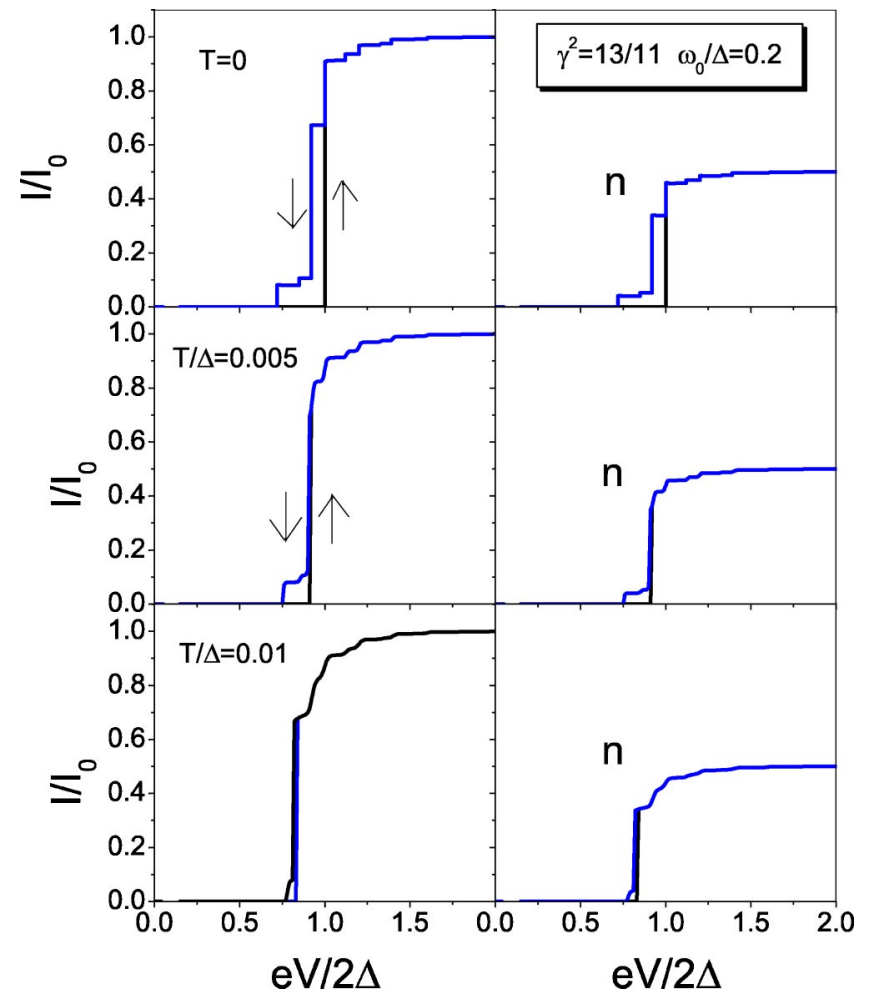

FIG. 4. (Color online) The I-V curves for tunneling through the molecular quantum dot with the electron-vibron coupling constant $\gamma^{2}=13 / 11$, which is slightly larger than that in Fig. 3. The I-V curves change substantially: the current pickup shifts to lower bias voltages and the curves show a substantial change of shape compared to Fig. 3. The hysteresis persists to slightly higher temperatures, although also small, as compared to the previous case.

peratures, the overall I-V curve shows the smoothed out steps at the bias voltages coinciding with the voltage at which the vibrons are emitted/absorbed, similar to the case of $T=0$ (Figs. 3 and 4).

\section{CONCLUSIONS}

In conclusion, we have developed the multipolaron theory of tunneling through a molecular quantum dot (MQD) taking phonon sidebands and strong electron correlations into account. The degenerate MQD with strong electron-vibron coupling shows a hysteretic volatile memory if the degeneracy of the molecular level is larger than two, $d>2$. The hysteretic behavior strongly depends on electron-vibron coupling and characteristic vibron frequencies. The current bistability vanishes above some critical temperature. It would be very interesting to look for an experimental realization of the model, possibly in a system containing a certain conjugated central part, which exhibits the attractive correlations of carriers with large degeneracy $d>2$. We have estimated earlier that $|U|$ is on the order of a few tenths on an $\mathrm{eV}$ in various oxide materials. ${ }^{32}$ Interaction may be of similar magnitude in carbon-based compounds. Interesting candidate 
systems are the $\mathrm{C}_{60}$ molecule $(d=6)$ where the electron-phonon interaction is strong, ${ }^{9}$ short nanotubes or other fullerenes $(d \gg 1)$, and mixed-valence molecular complexes. ${ }^{33}$ Switching should be fast, $10^{-13} \mathrm{~s}$ or less in carbon-based compounds.

\section{ACKNOWLEDGMENTS}

This work was partly supported by DARPA. The authors acknowledge useful discussions with V.V. Osipov and R.S. Williams.
${ }^{1}$ M. A. Kastner, Rev. Mod. Phys. 64, 849 (1992), and references therein.

${ }^{2}$ M. Büttiker, Phys. Rev. Lett. 57, 1761 (1986).

${ }^{3}$ P. W. Anderson, D. J. Thouless, E. Abrahams, and D. S. Fisher, Phys. Rev. B 22, 3519 (1980).

${ }^{4}$ P. A. Lee and D. S. Fisher, Phys. Rev. Lett. 47, 882 (1981).

${ }^{5}$ B. Shapiro, Phys. Rev. Lett. 48, 823 (1982).

${ }^{6}$ D. V. Averin, A. N. Korotkov, and K. K., Likharev, Phys. Rev. B 44, 6199 (1991).

${ }^{7}$ Y. Meir and N. S. Wingreen, Phys. Rev. Lett. 68, 2512 (1992).

${ }^{8}$ S. Hershfield, J. H. Davies, and J. W. Wilkins, Phys. Rev. B 46, 7046 (1992).

${ }^{9}$ J. Park, A. N. Pasupathy, J. I. Goldsmith, C. Chang, Y. Yaish, J. R. Retta, M. Rinkoski, J. P. Sethna, H. D. Abruña, P. L. McEuen, and D. C. Ralph, Nature (London) 417, 722 (2000).

${ }^{10}$ M. H. Hettler, H. Schoeller, and W. Wenzel, Europhys. Lett. 57, 571 (2002).

${ }^{11}$ J.-M. Lehn, Angew. Chem., Int. Ed. Engl. 29, 1304 (1990).

${ }^{12}$ J. M. Tour, Acc. Chem. Res. 33, 791 (2000); J. M. Tour et al., J. Am. Chem. Soc. 117, 9529 (1995).

${ }^{13}$ Molecular Electronics: Science and Technology, edited by A. Aviram and M. Ratner (Ann. N.Y. Acad. Sci., New York, 1998).

${ }^{14}$ C. P. Collier et al., Science 285, 391 (1999); J. Chen et al., ibid. 286, 1550 (1999); D. I. Gittins et al., Nature (London) 408, 677 (2000); H. X. He, T. J. Tao, L. A. Nagahara, I. Amlani, and R. Tsui, Phys. Rev. B (to be published).

${ }^{15}$ P. E. Kornilovitch, A. M. Bratkovsky, and R. S. Williams, Phys. Rev. B 66, 245413 (2002).

${ }^{16}$ P. E. Kornilovitch, A. M. Bratkovsky, and R. S. Williams, Phys. Rev. B 66, 165436 (2002).
${ }^{17}$ A. M. Bratkovsky and P. E. Kornilovitch, Phys. Rev. B 67, 115307 (2003).

${ }^{18}$ D. Stewart et al. (unpublished).

${ }^{19}$ A. S. Alexandrov, A. M. Bratkovsky, and R. S. Williams, Phys. Rev. B 67, 075301 (2003).

${ }^{20}$ N. B. Zhitenev, H. Meng, and Z. Bao, Phys. Rev. Lett. 88, 226801 (2002).

${ }^{21}$ L. I. Glazman and R. I. Shekhter, Zh. Éksp. Teor. Fiz. 94, 292 (1987) [Sov. Phys. JETP 67, 163 (1988)]; N. S. Wingreen, K. W. Jacobsen, and J. W. Wilkins, Phys. Rev. B 40, 11834 (1989).

${ }^{22}$ Xi Li, H. Chen, and S. X. Zhou, Phys. Rev. B 52, 12202 (1995).

${ }^{23}$ K. Kang, Phys. Rev. B 57, 11891 (1998).

${ }^{24}$ V. N. Ermakov, Physica E (Amsterdam) 8, 99 (2000).

${ }^{25}$ M. Di Ventra, S.-G. Kim, S. T. Pantelides, and N. D. Lang, Phys. Rev. Lett. 86, 288 (2001).

${ }^{26}$ H. Ness, S. A. Shevlin, and A. J. Fisher, Phys. Rev. B 63, 125422 (2001).

${ }^{27}$ U. Lundin and R. H. McKenzie, Phys. Rev. B 66, 075303 (2002).

${ }^{28}$ A. O. Gogolin and A. Komnik, cond-mat/0207513 (unpublished).

${ }^{29}$ In the case of buckminster fullerene anion $C_{60}^{-}$, Eq. (4) describes the coupling with $a_{g}$ modes, while the coupling with $h_{g}$ is off diagonal [for a detailed discussion, see I. D. Hands, J. L. Dunn, and C. A. Bates, Phys. Rev. B 63, 245414 (2001)].

${ }^{30}$ I. G. Lang and Yu. A. Firsov, Zh. Éksp. Teor. Fiz. 43, 1843 (1962) [Sov. Phys. JETP 16, 1301 (1963)].

${ }^{31}$ A. S. Alexandrov and N. F. Mott, Polarons and Bipolarons (World Scientific, Singapore, 1995).

${ }^{32}$ A. S. Alexandrov and A. M. Bratkovsky, Phys. Rev. Lett. 84, 2043 (2000).

${ }^{33}$ J. A. Wilson, J. Phys.: Condens. Matter 12, R517 (2000). 